\title{
Early dynamical evolution of star cluster systems
}

\author{
Geneviève Parmentier \\ Institute of Astrophysics \& Geophysics, Allée du 6 Août 17, B-4000 Liège, Belgium \\ Argelander Institut für Astronomie, Auf dem Hügel 71, D-53121 Bonn, Germany \\ email: gparm@astro.uni-bonn.de
}

\begin{abstract}
Violent relaxation, the protocluster dynamical response to the expulsion of its residual star-forming gas, is a short albeit crucial episode in the evolution of star clusters and star cluster systems. Because it is heavily driven by cluster-formation and environmental conditions, it is a potentially highly rewarding phase in terms of probing star formation and galaxy evolution. In this contribution, I review how cluster-formation and environmental conditions affect the shape of the young cluster mass function and the relation between the present star-formation rate of galaxies and the mass of their young, most massive cluster.
\end{abstract}

Keywords. stellar dynamics, stars: formation, galaxies: star clusters

\section{Introduction}

Compact, gas-embedded clusters are observed to be the formation sites of a significant fraction of stars in the local Universe. Following star formation, feedback processesranging from protostellar outflows, photoionization-driven gas overpressure and stellar winds to, eventually, supernovae - expel the gas that has not yet been converted into stars. That is, the efficiency with which a cluster parent core turns its gas into stars is always less than $100 \%$. Not only does gas expulsion terminate star formation, it also leaves the newly-formed cluster out of equilibrium, thereby forcing it into violent relaxation. In an attempt to regain a state of equilibrium, the cluster expands, loses a fraction of its stars or even undergoes complete disruption. These processes are referred to as cluster infant weight loss and infant mortality, respectively.

The dynamical response of clusters to gas expulsion implies that the cluster age distribution, the cluster mass function, the ratio of the mass in clusters and that in stars, as well as the distribution function of cluster half-mass radii all offer promising diagnostic tools of star cluster formation conditions. Baumgardt \& Kroupa (2007) have built and made available to the community an $N$-body model grid describing the temporal evolution of cluster mass loss and spatial expansion for a wide range of local star-formation efficiencies (SFEs; i.e., the mass fraction of dense molecular gas that cluster-forming cores turn into stars), gas-expulsion timescales and external tidal-field impacts. To date, this is the most comprehensive grid of model clusters through violent relaxation. Combined with the increasing quality of observational data sets (including of the much needed cluster half-mass radii: see, e.g., Scheepmaker et al.2007), the time is now ripe to decipher observed properties of young star clusters to probe their formation and environmental conditions.

The outline of this contribution is as follows. Following a brief reminder of the main parameters involved in violent relaxation in Section 2, I highlight how formation and environmental conditions affect the shape of the cluster mass function in Section 3. I also present some preliminary results of ongoing work regarding the relation between the 
luminosity of the brightest young cluster and the present star-formation rate (SFR) of spiral galaxies, the SFR $-M_{V}^{\text {brightest }}$ relation (Section 4).

\section{Gas expulsion: main parameters}

In what follows, the mass of a cluster is defined as the stellar mass enclosed within its tidal radius. We assume that the impact of the external tidal field is solely due to the smooth gravitational potential of a Milky Way-type galaxy, i.e., at this stage we neglect the potential impact on the early evolution of a star cluster of its natal giant molecular cloud. We adopt the Jacobi radius as an estimate of the cluster's tidal radius (see Baumgardt et al. 2009; their equation 1).

In the course of violent relaxation, the mass $m_{\mathrm{cl}}$ of a star cluster evolves rapidly and must therefore be defined as an instantaneous quantity. It obeys

$$
m_{\mathrm{cl}}=F_{\mathrm{b}}\left(t / \tau_{\text {cross }}, \varepsilon, \tau_{\mathrm{GExp}} / \tau_{\text {cross }}, r_{\mathrm{h}} / r_{\mathrm{t}}\right) \times \varepsilon \times m_{\mathrm{c}}
$$

where $m_{\mathrm{c}}$ is the mass of the progenitor molecular core, $\varepsilon$ the local SFE $\dagger$ and $F_{\mathrm{b}}$ the stellar mass fraction of the initially gas-embedded cluster still contained within the cluster tidal radius at time $t$. When using the term '(gas-)embedded cluster mass,' we refer to the stellar mass $m_{\mathrm{ecl}}=\varepsilon \times m_{\mathrm{c}}$. The local SFE is assumed to be uniform among all cores of a given simulation (i.e., no core-mass dependent SFE). The bound fraction, $F_{\mathrm{b}}$, depends on the time $t / \tau_{\text {cross }}$ elapsed since gas expulsion and on the gas-expulsion timescale, $\tau_{\mathrm{GExp}} / \tau_{\text {cross }}$ (both expressed in units of the molecular-core crossing time), on the local SFE, $\varepsilon$, and on the impact of the external tidal field. The latter is quantified as the ratio of the half-mass radius, $r_{\mathrm{h}}$, and the tidal radius, $r_{\mathrm{t}}$, of the gas-embedded cluster (see below for details). The older the cluster and/or the lower the SFE and/or the quicker gas expulsion and/or the stronger the host galaxy's tidal field, the lower $F_{\mathrm{b}}$. Building on the motion of a supershell propagating through the cluster-forming core and collecting the residual star-forming gas, Parmentier et al. (2008) derived an expression for the gas-expulsion timescale $\tau_{\mathrm{GExp}} / \tau_{\text {cross }}$ (their eq. 6 ; see also figure 3 in Parmentier \& Fritze 2009). Because a cluster expands following gas expulsion, the mass fraction of stars it loses depends on how compact the gas-embedded cluster is compared to its tidal radius. The impact of the external tidal field is therefore accounted for by the ratio $r_{\mathrm{h}} / r_{\mathrm{t}}$. As will be shown in Section 3, the cluster-forming core mass-radius relation is therefore of paramount importance for the outcome of violent relaxation, since it influences both the depth of the core potential well, and hence the gas-expulsion timescale, and the sensitivity to the external tidal field of the exposed cluster (see also Parmentier 2009).

\section{Young cluster mass functions}

In this section, I show how specific combinations of the core mass-radius relation, local SFE and external tidal field lead to cluster mass functions markedly different from the core mass function and, therefore, to mass-dependent cluster infant mortality. Cluster-forming cores are spatially resolved in the nearby regions of our Galaxy only, and the slope of the core mass-radius relation remains therefore poorly determined [see Parmentier et al. (submitted) for a discussion]. I consider two extreme cases in turn, i.e., constant core radii and constant core surface densities.

$\dagger$ We explicitly assume that stars have had sufficient time to reach virial equilibrium with the gas potential (i.e., gas expulsion occurs after a few crossing times) and, hence, that the local SFE closely matches the effective SFE (see Goodwin 2009 for a discussion). 


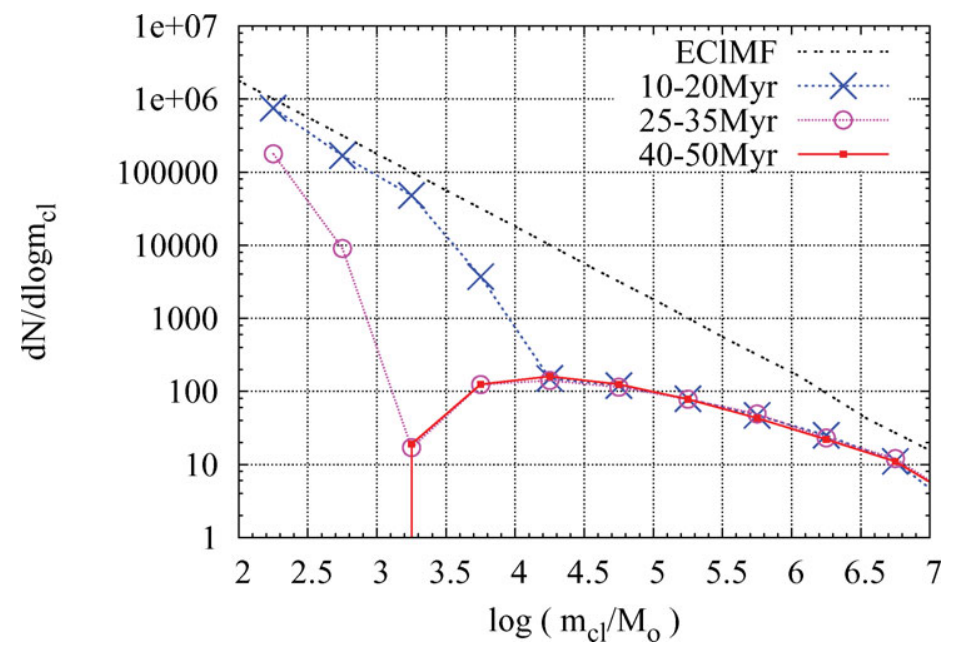

Figure 1. Time evolution of the cluster mass function for constant cluster-forming core radii $\left(r_{\mathrm{c}}=1 \mathrm{pc}\right)$ and the local SFE is $20 \%$. The symbol-free dotted (black) line is the power-law embedded-cluster mass function ('EClMF'). The lines with crosses, open circles and filled squares (blue, pink and red, respectively) show the cluster mass functions integrated over the age ranges quoted in the legend.

\section{Constant cluster-forming core radii and mass-varying gas expulsion timescale.}

Under the assumption of constant radii $\left(r_{\mathrm{c}}=1 \mathrm{pc}\right)$, a sequence of increasing core mass equates to one of increasing densities and deepening potential wells. As a result, high-mass cores undergo slower gas expulsion and retain a larger fraction of bound stars than their low-mass counterparts. Under the assumption of a weak tidal field, Parmentier et al. (2008) showed that the shape of the post-violent-relaxation cluster mass function is highly sensitive to the local SFE (see their figures 2 and 3 ). While a local SFE $\varepsilon \simeq 0.4$ is conducive to a cluster mass function mirroring the core mass function (mass-independent infant mortality and weight loss), a local SFE $\varepsilon \simeq 0.2$ results in the survival of the (initially) highest-mass embedded clusters only. For an exposed cluster to survive such a low local SFE, gas must be expelled adiabatically, a condition met by high-mass cores and their deep potential wells. Only those produce bound clusters at the end of violent relaxation, rendering cluster infant mortality heavily mass dependent. As a result, a power-law core-mass function gives rise to a bell-shaped cluster mass function.

An aspect which Parmentier et al. (2008) did not investigate is the time evolution of the power-law mass function of embedded clusters in the bell-shaped cluster mass function. The topic of evolving secularly (i.e., when violent relaxation effects have faded away) a power-law cluster mass function in a bell-shape is extensively covered in the literature. Starting from a power-law at an age of $\sim 50-100 \mathrm{Myr}$, internal two-body relaxation and external tidal stripping preferentially removes low-mass clusters, thereby carving a turnover in the mass function. As time goes by, this turnover moves towards higher cluster masses and smaller mass-function amplitudes. However, the evolution of relevance here is violent relaxation instead of secular evolution and, as we shall see, the cluster mass function evolves differently.

This is the perfect example to illustrate that the evolutionary stage of a cluster is not determined by its age in an absolute sense. As for the gas-expulsion timescale, what matters is the age expressed in units of core crossing times (see Equation 2.1). This implies that, keeping all other parameters the same, clusters born in cores of higher 


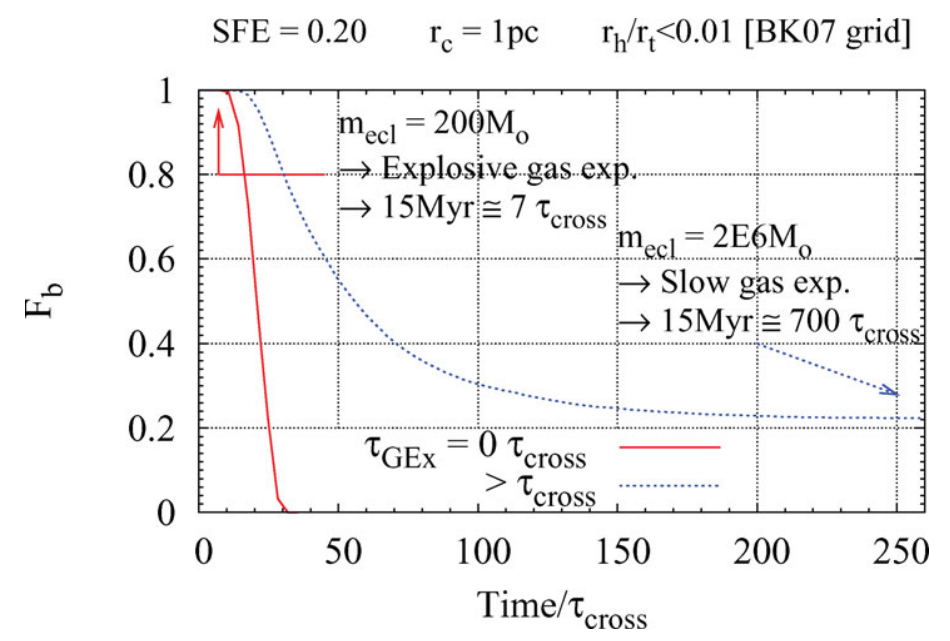

Figure 2. Time evolution of the bound fraction of stars for an SFE of $20 \%$ and two distinct regimes of cluster gas expulsion, i.e., explosive $\left(\tau_{\mathrm{GExp}} \ll \tau_{\text {cross }}\right)$ and adiabatic $\left(\tau_{\mathrm{GExp}}=3 \tau_{\text {cross }}\right)$. Under the assumption of constant core radii, high-mass clusters have a higher density, which results in slower gas expulsion, shorter crossing time and-once exposed-faster evolution than their low-mass counterparts.

densities evolve - once exposed - at a faster rate than those formed in low-density cores. Figure 1 shows the embedded-cluster mass function (i.e., the core-mass function shifted by -0.7 in $\log m$ to account for the constant $20 \%$ local SFE) and snapshots of the cluster mass function at ages of $\simeq 15,30$ and $45 \mathrm{Myr}$. The early cluster mass function (age $\simeq 15$ Myr; dotted line with $\times$ signs) shows prominent substructure, with cluster masses not yet significantly affected by infant weight loss in the low- and high-mass regimes $\left(m_{\mathrm{cl}}<3000 \mathrm{M}_{\odot}\right.$ and $m_{\mathrm{cl}}>2 \times 10^{6} \mathrm{M}_{\odot}$, respectively). They originate from how $F_{\mathrm{b}}$ evolves with time and as a function of gas-expulsion timescale. In Figure 2, the solid and dotted lines show the $F_{\mathrm{b}}$ time evolutions for explosive $\left(\tau_{\mathrm{GExp}} \ll \tau_{\text {cross }}\right)$ and adiabatic (here $\tau_{\text {GExp }}=3 \tau_{\text {cross }}$ ) gas expulsion, respectively. A massive embedded cluster (say, $m_{\mathrm{ecl}}=2 \times 10^{6} \mathrm{M}_{\odot}$ ) expels its residual gas on an adiabatic timescale and hence retains a bound core of stars in spite of the low SFE of $20 \%$. Also, by virtue of the high density of its natal core, a young age of $15 \mathrm{Myr}$ equates to several hundreds of core crossing times. Accordingly, cluster violent relaxation is over by this young age. This is why most of the bell shape, which arises from clusters initially more massive than $m_{\mathrm{ecl}} \sim 10^{6} \mathrm{M}_{\odot}$, is already carved by an age of $15 \mathrm{Myr}$.

By contrast, low-mass clusters (e.g., $m_{\mathrm{ecl}}=200 \mathrm{M}_{\odot}$ ) have low core densities, long core crossing times and the same age of $15 \mathrm{Myr}$ equates to a few $\tau_{\text {cross }}$ only, a stage at which the instantaneous bound fraction $F_{\mathrm{b}}$ is still close to unity. Consequently, the low-mass regime of the cluster mass function at an age of $15 \mathrm{Myr}$ sticks to the embedded-cluster mass function. As clusters age to $50 \mathrm{Myr}$, the cluster mass function in the high-mass regime does not evolve anymore and the low-mass regime of the mass function disappears as low-mass objects experience infant weight loss until their eventual dissolution.

\section{Constant cluster-forming core surface densities and mass-varying tidal-field impact.}

Following Parmentier et al. (2008), molecular cores of constant surface density have an (almost) constant gas-expulsion timescale. Yet, the infant-mortality and infant-weightloss rates of their embedded clusters are markedly mass dependent due to the tidal field impact. We insist that this effect takes place even if all cores are located within the same 


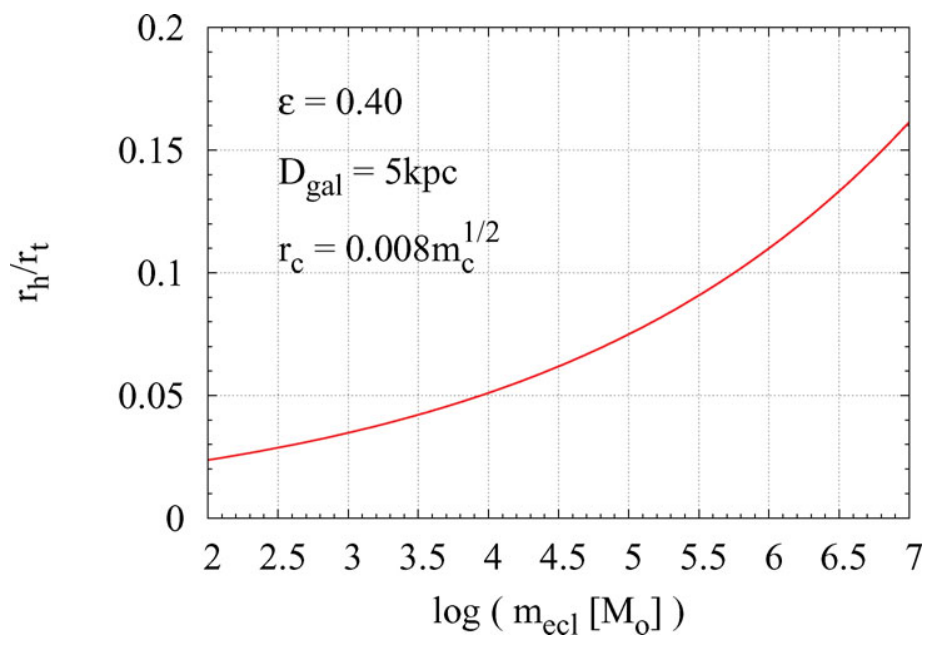

Figure 3. Dependence of the ratio of the half-mass radius, $r_{\mathrm{h}}$, and the tidal radius, $r_{\mathrm{t}}$ of the embedded cluster on its stellar mass, $m_{\mathrm{ecl}}$, for constant-surface-density cores. Because of their large spatial extent, high-mass clusters exhibit a greater sensitivity to the external tidal field and present a greater likelihood of disruption following gas expulsion.

limited region of a galaxy within which the external tidal field does not vary markedly. The ratio of the initial half-mass and tidal radii of these embedded clusters scales as $r_{\mathrm{h}} / r_{\mathrm{t}} \propto m_{\mathrm{c}}^{1 / 2} / m_{\mathrm{c}}^{1 / 3} \propto m_{\mathrm{c}}^{1 / 6}$ and, therefore, high-mass clusters have a greater sensitivity to the external tidal field since the volume of space in which they can expand freely is smaller than for low-mass clusters compared to the initial cluster size. This is illustrated in Figure 3, where the core mass-radius relation is $r_{\mathrm{c}}=0.008\left(m_{\mathrm{c}} / 1 \mathrm{M}_{\odot}\right)^{1 / 2} \mathrm{pc} \dagger$, the local SFE is $\varepsilon=0.4$ and the galactocentric distance is $D_{\text {gal }}=5 \mathrm{kpc}$ in a Milky Way-like potential. As a result, a power-law core-mass function evolves in a post-violent-relaxation cluster mass function depleted in high-mass objects (see Figure 4).

\section{What does it all mean?}

The key point to retain from this section is that the shape of the cluster mass function, the mass-radius relation of cluster-forming cores, their local SFE and the external tidal field are intimately linked issues. That most observed mass functions of young clusters are power laws with a spectral index of -2 [see Parmentier \& Gilmore (2007), and references therein; but see also Anders et al. (2007) for a possible turnover in the cluster mass function of the Antennae merger, NGC 4038/39] therefore tells us something about cluster formation conditions which are common to different types of cluster environments. The high sensitivity to the external tidal field of high-mass cores of constant surface density may be a hint that, contrary to theoretical expectations, the index $\beta$ of the mass-radius relation $r_{\mathrm{c}} \propto m_{\mathrm{c}}^{\beta}$ of cluster-forming cores is shallower than $\beta=1 / 2$. More simulations are required to settle this point conclusively. In particular, greater attention must be paid to cluster radius-related quantities, e.g., the distribution function of young cluster half-mass radii and the reason why clusters lack a clear mass versus half-mass radius correlation (Larsen 2004). We will investigate these aspects in a forthcoming paper.

$\dagger$ Core mass-radius relations adopted in this contribution are taken from Parmentier \& Fritze (2009). An update on these relations will be presented in Parmentier et al. (submitted). 


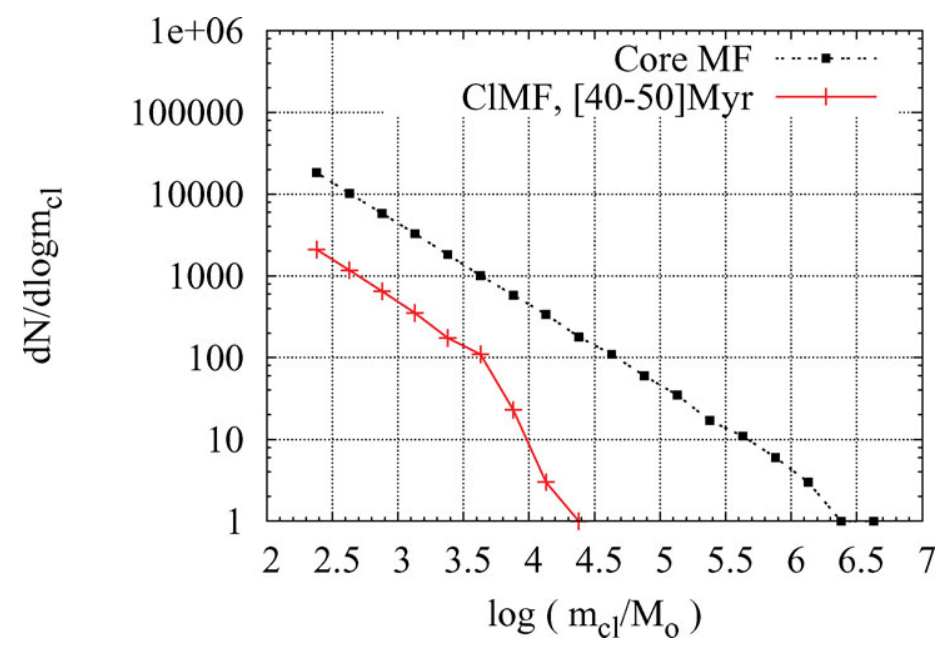

Figure 4. Constant-surface-density cores: when more massive objects are more vulnerable. The dotted (black) line is the core-mass function and the solid (red) line is the cluster mass function at an age of $\simeq 50 \mathrm{Myr}$. As can be understood from Figure 3, cluster infant mortality acts predominantly in the high-mass regime. Model parameters are the same as in Figure 3.

\section{Brightest young star clusters in galaxies}

Star clusters are chronometers of major star-formation events in their host galaxies (Kroupa 2002; Kotulla et al. 2008). When the SFR of a galaxy increases, not only does the galaxy form a larger number of gas-embedded star clusters, the cluster mass function is also sampled up to a higher cluster mass, partly as a result of the size-of-sample effect. Time spans in the history of galaxies characterized by vigorous star formation therefore show up prominently in cluster age histograms for two reasons: (i) the number of clusters is larger and (ii) these more numerous clusters also include more massive ones, which experience longer dissolution timescales and are therefore more likely to be observed.

Observationally, this effect is best known as a correlation between the present SFR of galaxies and the absolute visual magnitude, $M_{V}^{\text {brightest }}$, of their brightest cluster (Larsen 2002; Weidner et al. 2004; Bastian 2008). Building on synthetic cluster populations, Bastian (2008) shows that if galaxies form all their stars in gas-embedded clusters, a universal mass fraction of $8 \%$ of clusters survive the transition from their gas-embedded stage to being exposed.

That the ratio of the mass in clusters and stars depends sensitively on the mean local SFE (Parmentier \& Fritze 2009) prompted Parmentier et al. (submitted) to investigate which cluster-formation conditions are the main drivers of the SFR- $M_{V}^{\text {brightest }}$ relation. The absolute magnitude of a cluster depends on its age-dependent mass-to-light ratio, on the stellar mass of its parent gas-embedded cluster and on the amount of infant weight loss the latter has experienced. Section 3 demonstrates that cluster-mass-related quantities are heavily shaped by cluster-formation conditions. We have therefore divided our study of how the SFR- $M_{V}^{\text {brightest }}$ relation responds to input parameter variations into two main parts. First, we consider the limited age range of $10 \mathrm{Myr}$ over which we explicitly assume the constancy of the integrated cluster mass-to-light ratio, i.e., we investigate how the mass of the most massive cluster responds to cluster-formation conditions, specifically the core-mass function, core mass-radius relation, local SFE and strength of an external tidal field (Parmentier et al., submitted). In a second forthcoming paper, we will extend the cluster age range to $100 \mathrm{Myr}$ and assess how mass-to-light ratio variations, and hence 
Star formation efficiency in cluster-forming cores: 0.35

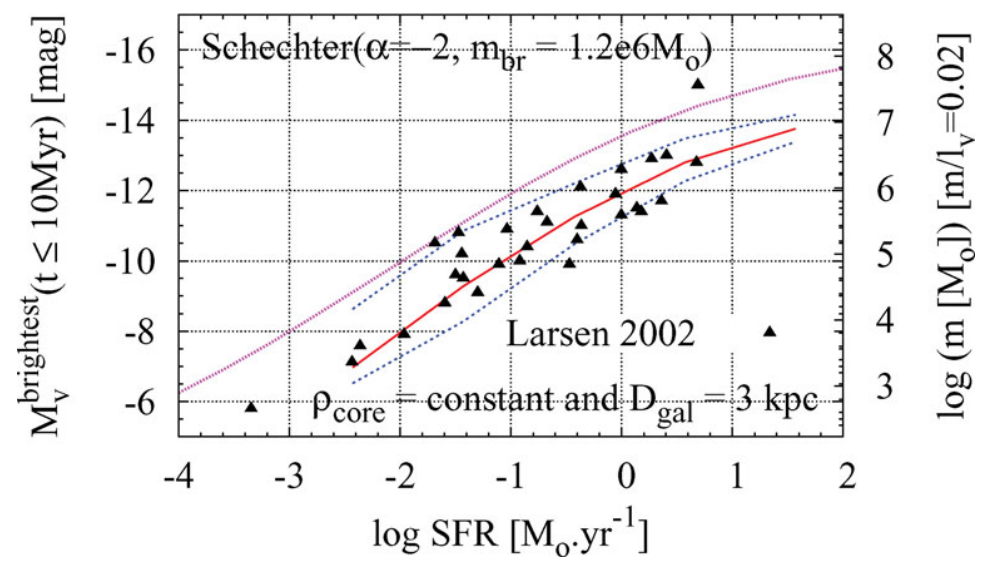

Figure 5. Observed relation between the absolute visual magnitude of the brightest cluster of spiral galaxies and their present SFR (filled triangles). The dotted (pink) line is the relation between the SFR and the mass of the most massive core for the adopted Schechter core-mass function $\left(\alpha=-2, m_{\mathrm{br}}=1.2 \times 10^{6} \mathrm{M}_{\odot}\right)$. Solid (red) and dashed (blue) lines represent an iso-SFE model and its $1 \sigma$ limits. We caution that, at the exploratory stage of this model, the evolutionary time span of the model $(10 \mathrm{Myr})$ is narrower than that of the observations ( $\gtrsim 100 \mathrm{Myr})$, thereby preventing us from drawing conclusions about the best local SFE estimate (see text for details).

the age of the observed brightest cluster, contribute to shape the $\mathrm{SFR}-M_{V}^{\text {brightest }}$ relation. As for the first part of the study, our findings are as follows. The vertical location of the SFR $-M_{V}^{\text {brightest }}$ relation depends on the external tidal field and local SFE. Stronger tidal fields and/or smaller SFE are conducive to smaller mass/brightness for the most massive/brightest clusters. As for the shape of the SFR $-M_{V}^{\text {brightest }}$ relation, it is dictated mostly by the core-mass function (see Figure 5) and the core mass-radius relation. For constant-surface-density cores, the $\mathrm{SFR}-M_{V}^{\text {brightest }}$ relation becomes shallower when the SFR increases because of the greater sensitivity to the external tidal field of high-mass embedded clusters and hence the larger amount of infant weight loss they experience. By contrast, for constant core radii, it becomes steeper towards higher SFR as, in this case, higher-mass cores experience slower gas expulsion and thus retain a larger bound fraction of stars.

In the case illustrated in Figure 5, cluster-forming cores have a constant volume density $\left(r_{\mathrm{c}}=0.026\left(m_{\mathrm{c}} / 1 \mathrm{M}_{\odot}\right)^{1 / 3} \mathrm{pc}\right)$, the local SFE is $35 \%$ and the synthetic cluster population is located at a galactocentric distance of $3 \mathrm{kpc}$ in a Milky Way-like potential. The coremass function is a Schechter function with a spectral index of -2 and its break mass, $m_{\mathrm{br}}$, has been adjusted so that, following star formation and infant weight loss, the break mass of the cluster mass function is about $\simeq 2 \times 10^{5} \mathrm{M}_{\odot}$, as observed for spiral galaxies (Larsen 2009). The solid and dotted lines are the model and its $1 \sigma$ limits, respectively. The filled triangles are the data points from Larsen (2002) for spiral galaxies. Figure 5 suggests that an iso-SFE model provides a good fit to the ensemble of the data, provided that other conditions - core mass-radius relation (its slope and normalization), external tidal field - do not vary significantly from one spiral galaxy to another. We emphasize once again that the simulation time span $(10 \mathrm{Myr})$ is shorter than the age range of clusters shown in this plot $(\gtrsim 100 \mathrm{Myr})$. Since the model vertical location is also age dependent through both the amount of infant weight loss and the mass-to-light ratio, the limited time span of our simulations prevents us from drawing firm conclusions about 
the best local SFE estimate. Having now obtained a firm handle on how cluster formation and environmental conditions affect the relation between the present SFR and the mass of the young, most massive cluster, we are ready to perform simulations on a larger scale, encompassing $\simeq 100 \mathrm{Myr}$ of dynamical evolution and in which mass-to-light ratio variations will be taken into account. This will give us a lower limit to the local SFE in gas-embedded clusters in spiral galaxies. The actual value will depend on the importance of the distributed mode of star formation.

\section{Acknowledgements}

I am grateful to the Scientific Organising Committee of IAU Symposium 266 for their invitation to deliver this talk and to the IAU for financial support. Support from the Belgian Science Policy Office in the form of a Return Grant and from the Alexander von Humboldt Foundation in the form of a Research Fellowship are acknowledged.

\section{References}

Anders, P., Bissantz, N., Boysen, L., de Grijs, R., Fritze-v. Alvensleben, U. 2007, MNRAS, 377, 91

Bastian, N. 2008, MNRAS, 390, 759

Baumgardt, H. \& Kroupa P. 2007, MNRAS, 380, 1589

Baumgardt, H., Parmentier, G., Gieles, M., \& Vesperini E., 2009, MNRAS, in press (arXiv:0909.5696)

Goodwin, S. P. 2009, in: E. Pérez, R. de Grijs \& R. M. González Delgado, Young massive stars clusters - Initial conditions and environments, ApSS, 324, 259

Kotulla, R., Fritze, U., \& Anders, P. 2008, MNRAS, 387, 1149

Kroupa, P. 2002, MNRAS, 330, 707

Larsen, S. S. 2002, AJ, 124, 1393

Larsen, S. S. 2004, $A \mathscr{E} A, 416,537$

Larsen, S. S. 2009, $A \mathscr{E} A, 494,539$

Parmentier, G. \& Gilmore, G. 2007, MNRAS, 377, 352

Parmentier, G., Goodwin, S., Kroupa, P., \& Baumgardt, H. 2008, ApJ, 678, 347

Parmentier, G. \& Fritze, U. 2009, ApJ, 690, 112

Parmentier, G. 2009, Rev. Mod. Astron., 21, in press (arXiv:0901.3140)

Parmentier, G., Kroupa, P., \& Baumgardt, H., ApJ, submitted

Scheepmaker, R. A., Haas, M. R., Gieles, M., Bastian, N., Larsen, S. S., \& Lamers, H. J. G. L. M. 2007, $A \& B A, 469,925$

Weidner, C., Kroupa, P., \& Larsen, S. S. 2004, MNRAS, 350, 1503 